\title{
Supplementary Material: Bayesian Estimation of Correlation Matrices of Longitudinal Data
}

\author{
Riddhi Pratim Ghosh, Bani Mallick, and Mohsen Pourahmadi
}

\section{Appendix A: Proof of Theorem 1}

Before proving Theorem 1, we state following two useful propositions required for the proof.

Proposition 1. The correlation coefficient $r_{i j}$ is related to the semi-partial correlations via

$$
\begin{aligned}
& r_{i j}=\rho_{j i: 1,2, \ldots, j-1} \prod_{u=1}^{j-1} \sqrt{1-\rho_{u j: 1,2, \ldots, u-1}^{2}} \sqrt{1-\rho_{u i: 1,2, \ldots, u-1}^{2}} \\
& \left.+\sum_{u=1}^{j-1}\left(\rho_{u j: 1,2, \ldots, u-1} \prod_{m=1}^{u-1} \sqrt{1-\rho_{m j: 1,2, \ldots, m-1}^{2}}+\rho_{u i: 1,2, \ldots, u-1} \prod_{m=1}^{u-1} \sqrt{1-\rho_{m i: 1,2, \ldots, m-1}^{2}}\right) \text { for } 1 \leq j<i \leq k .1\right)
\end{aligned}
$$

Proof. The proof is based on the following recurrence relation for partial correlations (Lewand bwski et al., 2009),

$$
\rho_{j i: k, L}=\frac{\rho_{j i: L}-\rho_{k i: L} \rho_{k j: L}}{\sqrt{\left(1-\rho_{k i: L}^{2}\right)\left(1-\rho_{k j: L}^{2}\right)}}
$$

where $L$ is a possibly empty set of indices with $i, j, k \notin L$. Using the above recurrence relation repeatedly, one notes that

$$
\begin{aligned}
& r_{j i}=\rho_{j i}=\rho_{j i: 1} \sqrt{\left(1-\rho_{1 j}^{2}\right)\left(1-\rho_{1 i}^{2}\right)}+\rho_{1 j} \rho_{1 i} \\
& \rho_{j i: 1}=\rho_{j i: 1,2} \sqrt{\left(1-\rho_{2 j: 1}^{2}\right)\left(1-\rho_{2 i: 1}^{2}\right)}+\rho_{2 j: 1} \rho_{2 i: 1} \\
& \text { Therefore, } r_{j i}=\rho_{j i: 1,2} \sqrt{\left(1-\rho_{2 j: 1}^{2}\right)\left(1-\rho_{2 i: 1}^{2}\right)\left(1-\rho_{1 j}^{2}\right)\left(1-\rho_{1 i}^{2}\right)}+\rho_{2 j: 1} \rho_{2 i: 1} \rho_{1 j} \rho_{1 i}
\end{aligned}
$$

which is conformable with S.1. One can further substitute $\rho_{j i: 1,2}$ by terms involving $\rho_{j i: 1,2,3}$ and using these repeatedly the result follows. 
Proposition 2. If $b_{m, j}=\rho_{j m: 1,2, \ldots, j-1} \prod_{u=1}^{j-1} \sqrt{1-\rho_{u m: 1,2, \ldots, u-1}^{2}}$, then

$$
1-\sum_{u=1}^{j-1} b_{j u}^{2}=\prod_{u=1}^{j-1}\left(1-\rho_{u j: 1,2, \ldots, u-1}^{2}\right)
$$

Proof. We first note that number of terms in left-hand side in $(\mathrm{S.2})$ and right-hand side in is $j$. Then the proof will be complete by comparing the coefficient of $\rho_{m j: 1,2, \ldots m-1}^{2}$ on both sides in $\mathrm{S.2}$ where $m$ can take any value in $\{1,2, \ldots, j-1\}$.

Note that $\rho_{m j: 1,2, \ldots, m-1}^{2}$ enters in left-hand side of $\mathrm{S.2}$ only through $b_{j m}^{2}$ and thus its co-

efficient equals to $-\prod_{u=1}^{m-1} \sqrt{1-\rho_{u m: 1,2, \ldots, u-1}^{2}}$ which is also the coefficient of $\rho_{m j: 1,2, \ldots, m-1}^{2}$ in right-hand side.

\section{Proof of Theorem 1}

(a) Note that $r_{i 1}=b_{i 1} b_{11}$ and $b_{11}=1$. Thus, $r_{i 1}=b_{i 1}$ for $i=2,3, \ldots, k$.

For $i=j, r_{i i}=1=\sum_{u=1}^{i} b_{i u} b_{i u}$. Therefore, $b_{i i}=\sqrt{1-\sum_{u=1}^{i-1} b_{i u}^{2}}$.

To prove the form of $b_{i j}$ for $2 \leq j<i \leq k$ in (2), we resort to induction on dimension ( $k$ ) of $R$, where induction step starts from $k=3$.

In this case, $r_{32}=b_{31} b_{21}+b_{32} b_{22}$. Hence, $b_{32}=\frac{r_{32}-b_{31} b_{21}}{b_{22}}=\frac{r_{32}-r_{31} r_{21}}{\sqrt{1-r_{21}^{2}}}=\rho_{32: 1} \sqrt{1-r_{31}^{2}}$, which satisfies (2).

Suppose (2) holds for any correlation matrix of dimension $k$. We require to prove it for a $(k+1)$ dimensional correlation matrix $R_{k+1}$. We write

$$
R_{k+1}=\left[\begin{array}{cc}
R_{k} & r_{k+1} \\
r_{k+1}^{\mathrm{T}} & 1
\end{array}\right]
$$

where $R_{k}$ is the $k$-dimensional correlation matrix pertaining to first $k$ variables and $r_{k+1}^{\top}=$ $\left(r_{k+1,1}, r_{k+1,2}, \ldots, r_{k+1, k}\right)$

Let $R_{k+1}=B_{k+1} B_{k+1}^{\top}$ be the Cholesky decomposition of $R_{k+1}$, where $B_{k+1}$ is a lower triangular matrix. Then $B_{k+1}^{\top}=\left[B_{k}^{\top}: \lambda_{k+1}^{\top}\right]^{\top}$, where $B_{k}$ is the lower triangular Cholesky factor of $R_{k}$. In the context of induction hypothesis, we only require to show that elements of $\lambda_{k+1}$ are in the form of (2), where we assume elements of $B_{k}$ are already in the form of (2). We note that $\lambda_{k+1,1}=r_{k+1,1}$ and equating $r_{k+1, j}$ from the Cholesky decomposition one 
gets,

$$
\begin{aligned}
& \lambda_{k+1, j}=\frac{r_{k+1, j}-r_{j 1} r_{i 1}-\sum_{u=1}^{j-1} b_{j u} \lambda_{k+1, u}}{\sqrt{1-\sum_{u=1}^{j-1} b_{j u}^{2}}} \\
& =\frac{r_{k+1, j}-r_{j 1} r_{i 1}-\sum_{u=1}^{j-1} \rho_{u j: 1,2, \ldots, u-1} \rho_{u, k+1: 1,2, \ldots, m-1} \prod_{m=1}^{u-1} \sqrt{\left(1-\rho_{m, k+1: 1,2, \ldots, m-1}^{2}\right)\left(1-\rho_{m j: 1,2, \ldots, m-1}^{2}\right)}}{\prod_{u=1}^{j-1} \sqrt{1-\rho_{u j: 1,2, \ldots, u-1}^{2}}} \\
& =\frac{\rho_{j, k+1: 1,2, \ldots, j-1} \prod_{u=1}^{j-1} \sqrt{\left(1-\rho_{u j: 1,2, \ldots, u-1}^{2}\right)\left(1-\rho_{u, k+1: 1,2, \ldots, u-1}^{2}\right)}}{\prod_{u=1}^{j-1} \sqrt{1-\rho_{u j: 1,2, \ldots, u-1}^{2}}} \\
& =\rho_{j, k+1: 1,2, \ldots, j-1} \prod_{u=1}^{j-1} \sqrt{1-\rho_{u, k+1: 1,2, \ldots, u-1}^{2}}
\end{aligned}
$$

where second and third steps follow from induction hypothesis, S.1) and (S.2).

(b) Since the diagonal entries of the matrix $B$ are non-negative, the statement follows from the representation of the Cholesky factor $B$ and the uniqueness of the Cholesky factor of $R$. Consequently, the angles $\theta_{i j}$ 's are simply the inverse cosine of the semi-partial correlations, and as such they are readily interpretable statistically.

\section{Appendix B: Proof of Theorem 2}

Note that $\operatorname{det}(R)=\operatorname{det}\left(B B^{\top}\right)=(\operatorname{det}(B))^{2}=\prod_{k=1}^{k-1} \prod_{i=j+1}^{k} \sin ^{2} \theta_{i j}$, and the Jacobian of the transformation is $\left|\operatorname{det}\left(\frac{\partial R}{\partial \Theta}\right)\right|=\prod_{j=1}^{k}\left(\prod_{i=j+1}^{k+1} \sin \theta_{i j}\right)^{k+1-j}$. Then the joint distribution of $R$ is

$$
\begin{aligned}
& p(R)=p(\Theta) \times\left|\operatorname{det}\left(\frac{\partial \Theta}{\partial R}\right)\right|=\frac{\prod_{j=1}^{k-1} \prod_{i=j+1}^{k} p_{j}\left(\theta_{i j}\right)}{\left|\operatorname{det}\left(\frac{\partial R}{\partial \Theta}\right)\right|} \\
& =\frac{\prod_{j=1}^{k-1} \prod_{i=j+1}^{k} c_{j} \times\left(\sin \theta_{i j}\right)^{2 \alpha+k-j}}{\prod_{j=1}^{k-1}\left(\prod_{i=j+1}^{k} \sin \theta_{i j}\right)^{k-j}} \\
& =\prod_{j=1}^{k-1} \prod_{i=j+1}^{k} c_{j} I\left(\theta_{i j} \in[0, \pi)\right) \times\left(\prod_{j=1}^{k-1} \prod_{i=j+1}^{k} \sin ^{2} \theta_{i j}\right)^{\alpha} \\
& =c_{k}(\alpha)(\operatorname{det}(R))^{\alpha}
\end{aligned}
$$


where the normalizing constant

$$
\begin{aligned}
& c_{k}(\alpha)=\frac{1}{\prod_{j=1}^{k-1}\left(\int_{0}^{\pi} \sin ^{2 \alpha+j}(\theta) d \theta\right)^{j}} \\
& =\frac{1}{\prod_{j=1}^{k-1}\left(B\left(\frac{2 \alpha+j+1}{2}, \frac{1}{2}\right)\right)^{j}} \text { (where } B \text { is the Beta function) } \\
& =\prod_{j=1}^{k-1}\left(\frac{\Gamma\left(\frac{2 \alpha+j}{2}+1\right)}{\Gamma\left(\frac{2 \alpha+j+1}{2}\right) \Gamma\left(\frac{1}{2}\right)}\right)^{j}=\prod_{j=1}^{k-1}\left(\frac{\Gamma\left(\frac{2 \alpha+j}{2}+1\right)}{\sqrt{\pi} \Gamma\left(\frac{2 \alpha+j+1}{2}\right)}\right)^{j}
\end{aligned}
$$

and hence the proof of part(a).

For part(b), note that the distribution of $R$ is invariant under permutation of rows and columns. Thus it is enough to show that the distribution of $r_{k, 1}$ is $\operatorname{Beta}(\alpha+k / 2, \alpha+k / 2)$. The distribution of $r_{k, 1}$ is

$$
p\left(r_{k, 1}\right) \propto\left(\sin (\arccos (r))^{2 \alpha+k-1}\right) \frac{d \arccos (r)}{d r} \propto\left(1-r^{2}\right)^{\alpha+k / 2-1} .
$$

Part(c) is trivial since $\sin \theta$ is symmetric in $[0, \pi)$ having mode at $\pi / 2$.

\section{References}

Lewandowski, D., Kurowicka, D., \& Joe, H. (2009). Generating random correlation matrices based on vines and extended onion method. Journal of Multivariate Analysis, 100(9), 1989-2001. 